\title{
Solar image segmentation by use of mean field fast annealing
}

\author{
E. Bratsolis ${ }^{1,2}$ and M. Sigelle ${ }^{3}$ \\ 1 Astronomical Institute, National Observatory of Athens, P.O. Box 20048, GR-11810 Athens, Greece \\ 2 Section of Astrophysics, Astronomy and Mechanics, Department of Physics, University of Athens, GR-15784 Athens, Greece \\ 3 École Nationale Supérieure des Télécommunications, Département Images, 46 rue Barrault, F-75634 Paris Cedex 13, France
}

Received September 8, 1997; accepted February 23, 1998

\begin{abstract}
We present a "continuous" analysis of a solar $\mathrm{H}_{\alpha}$ image in order to address the problem of image segmentation. Our approach is based on combinatorial optimization methods and in particular on Mean Field Fast Annealing (MFFA). Mean-field theory gives a deterministic nature to our algorithm while its efficiency is improved by a fast cooling schedule. We show how this method can be used to separate efficiently the regions of different solar activity giving a tool for a future automated recognition and classification of sunspots.
\end{abstract}

Key words: sunspots — methods: data analysis

\section{Introduction}

Sunspots and sunspot groups are the most conspicuous features of solar activity. They appear as dark areas in the photosphere of the sun, with sizes and numbers that vary in the 11-year cycle and they are caused by a lower surface temperature.

The area covered by the sunspot is one of the most relevant parameters used to investigate the solar activity since the sunspot number is only an empirical index. In many occasions a phase delay between the value of the sunspot number and the projected areas of sunspots has been observed (e.g. Pap 1985). There is some recent evidence pointing to a relation between magnetic field strength, continuum intensity and sunspot size (Collados et al. 1994). The ratio of the sunspot and facular areas is linked with the age of the active regions (young or old sunspot groups) (Pap 1985). The energy balance of individual active regions seems to depend mainly on the area ratio of the plage and the corresponding sunspot group (Steinegger et al. 1996). During the solar minimum, when only a few active regions exist on the Sun, the main contribution to the irradiance variations arises from the active network, which is formed by

$\overline{\text { Send offprint requests to }}$ : E. Bratsolis (ebrats@atlas.uoa.gr) the breakup and dispersion of the active regions (Fröhlich \& Pap 1989).

The determination of sunspot area becomes more complicated when one attempts to separate the individual areas of umbra and penumbra. Steinegger et al. (1997) compare two methods of measuring sunspot areas from photospheric observations; one based on histogram modifications and a new method termed inflexion point method (IPM).

Therefore, it is of great importance to isolate the basic characteristics of the sunspot or sunspot group, in order to compare them with active regions and to study the results of solar activity. In $\mathrm{H}_{\alpha}$, the chromosphere is particularly bright and the matter is apparently dominated by the chromospheric magnetic field lines.

The purpose of segmentation is to divide the image into meaningful regions that correspond to structural units in the scene or distinguish objects of interest. In this case it could be useful if one wants to isolate automatically the basic characteristics of the sunspot or sunspot group and study their relation with solar activity using $\mathrm{H}_{\alpha}$ observations.

The segmentation is separated here in two stages, the classification in $q$ labels (Sect. 2) and the relaxation labeling process (Sect. 3).

\section{Observations and data reduction}

The $\mathrm{H}_{\alpha}$ observations used in this work were taken at Sacramento Peak Observatory on December 14, 1996. Our image (Fig. 1) has dimensions $128 \times 128$ pixels with 42 different intensities. The minimum value is 32 and the maximum is 222 . The resolution is $0.4 \mathrm{arcsec} / \mathrm{pixel}$ and thus our image contains an area of $51.2 \times 51.2 \operatorname{arcsec}^{2}$. As a first step we sharpen this image to enhance the local contrast by convolving it with the following template:

$\left(\begin{array}{ccc}-1 & -2 & -1 \\ -2 & 13 & -2 \\ -1 & -2 & -1\end{array}\right)$ 


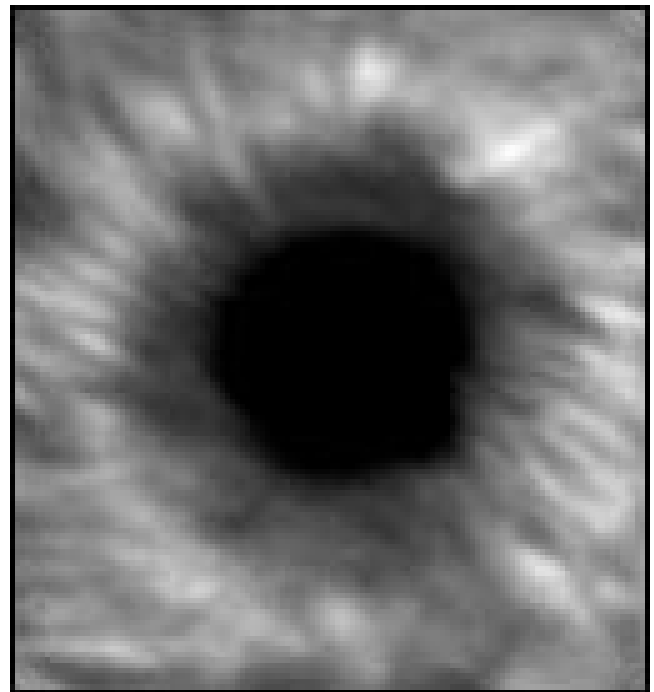

Fig. 1. Selected sunspot image $(128 \times 128)$ containing an area of $51.2 \times 51.2 \operatorname{arcsec}^{2}$

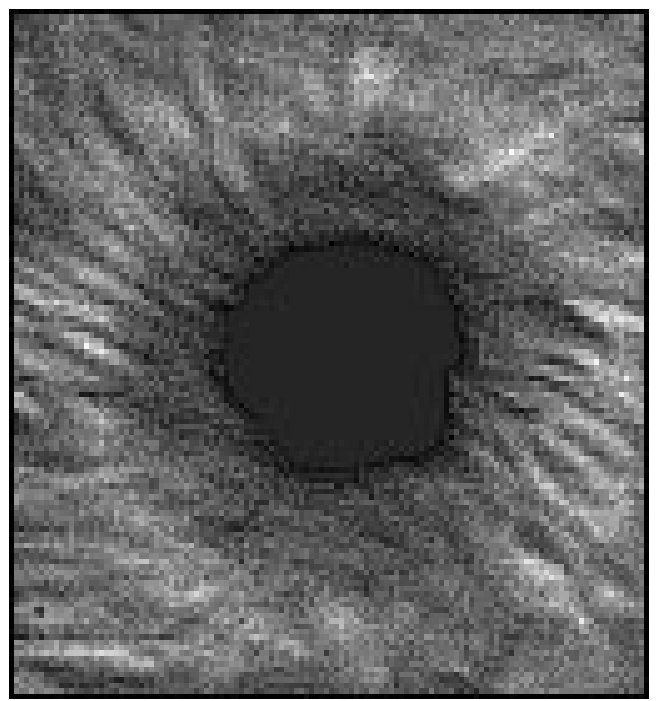

Fig. 2. Sunspot image $(128 \times 128)$ after sharpening of Fig. 1 with template of Sect. 2

The new image (Fig. 2) has a minimum of -20 and a maximum of 330 . Let $I(x, y)$ be our image matrix of pixel intensities of $(x, y)$ (Fig. 1). The image after sharpening (Fig. 2) has intensities $I_{1}(x, y)$. Let now $\mathcal{M}=\max \left(I_{1}(x, y)\right)$ and $m=\min \left(I_{1}(x, y)\right)$. We reduce our image (Fig. 2) into $q$ classes (Figs. 3, 4) using the following equation:

$I_{2}(x, y)=\operatorname{int}\left(\frac{I_{1}(x, y)-m}{\mathcal{M}-m}(q-1)\right)$.

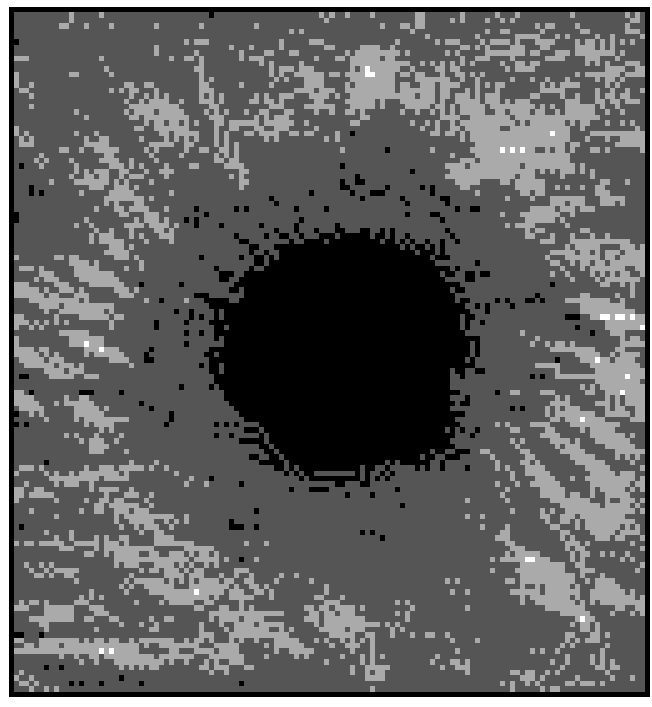

Fig. 3. Sunspot image $(128 \times 128)$ after application of Eq. (1) in Fig. 2 with $q=4$ labels

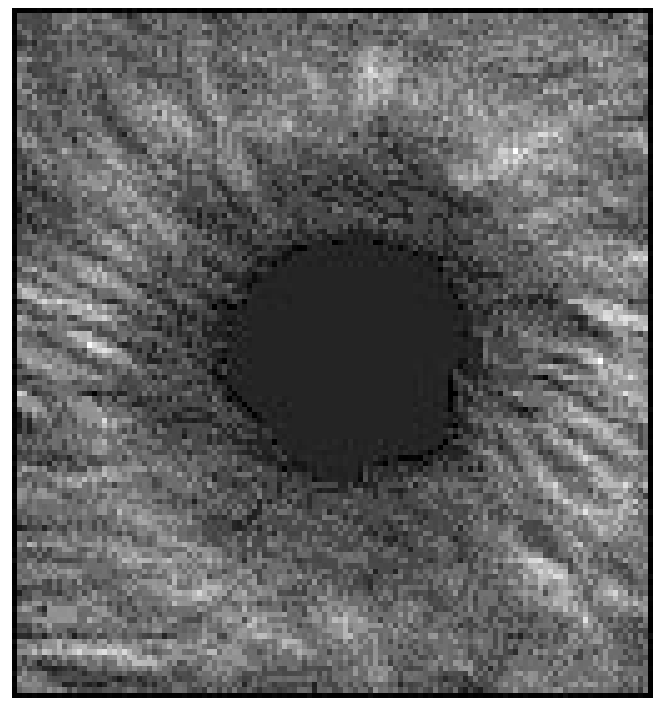

Fig. 4. Sunspot image $(128 \times 128)$ after application of Eq. (1) in Fig. 2 with $q=8$ labels

\section{Relaxation labeling process}

Our sunspot image is taken at the center of $\mathrm{H}_{\alpha}$. Our purpose is to divide the image into regions of different activity (e.g., umbra, penumbra, active regions) and therefore the problem now is reduced in a relaxation labeling process. Relaxation labeling processes were originally developed to reduce ambiguity and noise and select the best label among several possible choices in vision systems (Hummel \& Zucker 1983). We consider it as a particular case of combinatorial optimization problems (e.g., Titterington 1985; Jeffrey \& Rosner 1986) for which the cost function corresponds to the global energy of a complex physical 
system (e.g., Carnevali et al. 1985; Hiriyannaiah et al. 1989; Bilbro et al. 1991; Hérault \& Horaud 1993; Bratsolis \& Sigelle 1997).

\subsection{Potts interaction}

Let $\mathcal{I}=\{0,1, \ldots q-1\}$ be the set of gray levels (labels or classes). A label random variable $l_{s}$ is associated for each site $s \in S=\{1,2 \ldots N\}$, where $N$ is the number of pixels. The true distribution of $l_{s}$ is unknown, but based on some measurements, a probability distribution $P\left(l_{s}\right): \mathcal{I} \mapsto[0,1]$ is estimated for such a random variable.

Real images are often inhomogeneous, with nearly uniform regions separated by relatively sharp edges. To get round this problem, local energy functions have been introduced. To express the local properties of an image, a neighborhood system of $r$-pixels is defined as a collection of pixels that are assumed to interact directly with $s$-pixel. A basic characteristic of most images is that intensity values at neighbor locations $r$ and $s$ are likely to be similar (e.g., Besag 1974; Besag 1986).

The Hamiltonian of our system is the cost function of the lattice. Using some ideas from Statistical Physics, we can use a Potts potential interaction model $U_{r s}(\mathrm{Wu} 1982$; Bratsolis \& Sigelle 1997).

$U_{r s}=-K \frac{q-1}{q}\left(\hat{u}_{s} \cdot \hat{u}_{r}\right)-\frac{K}{q}+\frac{K}{2}$

where $K$ is a constant and $\hat{u}_{s}, \hat{u}_{r}$ random (unit) vectors at sites $s, r$ under a $(q-1)$-dimensional space with values in $R$.

\subsection{Mean-field theory}

One of the basic ideas of the mean-field approximation, is to find the best linear approximation, in a statistical sense, of a Hamiltonian expression. So, Eq. (2) is written up to a constant,

$$
\begin{gathered}
U_{r s}=-\beta\left\{\hat{u}_{s} \cdot E\left(\hat{u}_{r}\right)+\hat{u}_{r} \cdot E\left(\hat{u}_{s}\right)-E\left(\hat{u}_{s}\right) \cdot E\left(\hat{u}_{r}\right)\right. \\
\left.+\left[\hat{u}_{s}-E\left(\hat{u}_{r}\right)\right] \cdot\left[\hat{u}_{r}-E\left(\hat{u}_{s}\right)\right]\right\}
\end{gathered}
$$

where $\beta=K \frac{q-1}{q}, E\left(\hat{u}_{s}\right)$ the expected value of vector $\hat{u}_{s}$. and $E\left(\hat{u}_{r}\right)$ the expected value of vector $\hat{u}_{r}$. Using mean-field theory, we consider only the first two terms of Eq. (3). Each local energy function is thus approximated by a sum of first-order clique potentials, leading to a quasi-independent vector approximation.

The total energy of the Potts model with mean-field approximation up to a constant is:

$U=-\beta \sum_{(s, r)} \hat{u}_{s} \cdot \hat{u}_{r} \approx-\beta \sum_{s \in S} \hat{u}_{s} \cdot\left[\sum_{r \in N_{S}} E\left(\hat{u}_{r}\right)\right]$ or

$U=-\sum_{s \in S} \mathbf{h}_{s} \cdot \hat{u}_{s}$

where $\mathbf{h}_{s}=\beta \sum_{r \in N_{S}} E\left(\hat{u}_{r}\right)$ is the effective magnetic field (in physical sense) produced by neighbors of site $s$ and $N_{s}$ the set of the $r$-neighbors of the site $s$. There is no link between the effective magnetic field $\mathbf{h}_{s}$ and sunspot magnetic field.

Calling now $\mathcal{N}_{s}$ the configuration of these neighbor sites, we have

$E\left(\hat{u}_{s}\right)=\sum_{k=0}^{q-1} P\left(\hat{u}_{s}=\hat{v}_{k}\right) \hat{v}_{k}=\sum_{k=0}^{q-1} P\left(\hat{u}_{s}=\hat{v}_{k} / \mathcal{N}_{s}\right) \hat{v}_{k}$.

Here $P\left(\hat{u}_{s}=\hat{v}_{k} / \mathcal{N}_{s}\right)$ is simultaneously the local and global Gibbs-Boltzmann distribution $\hat{u}_{s}$, on account of our assumption of vector variables independence. The expected value of vector $\hat{u}_{s}$ at site $s$ is then computed as

$E\left(\hat{u}_{s}\right)=\frac{\sum_{k=0}^{q-1} \hat{v}_{k} \exp \left[\frac{\beta}{T} \sum_{r \in N_{s}} \hat{v}_{k} \cdot E\left(\hat{u}_{r}\right)\right]}{\sum_{k=0}^{q-1} \exp \left[\frac{\beta}{T} \sum_{r \in N_{s}} \hat{v}_{k} \cdot E\left(\hat{u}_{r}\right)\right]} \forall s \in S$

Equation (7) gives rise to a self-consistent nonlinear dynamical system of $q$ equations, where $T>0$ is a parameter at our disposal and, in physical terms, represents the "absolute temperature" of the system. The strict relationship between Markov random-field hypothesis is evident. An extended mathematical description of Potts interaction with mean-field approximation is in Bratsolis \& Sigelle (1997). The mean-field equations in a probabilistic interpretation in iterative form is given by:

$$
\begin{aligned}
& {\left[P\left(l_{s}=i\right)\right]^{\text {new }} } \\
= & \frac{\exp \left(\frac{1}{T}\left\{\sum_{r \in N_{s}} K_{i i}^{r s}\left[P\left(l_{r}=i\right)\right]^{\text {old }}+B \delta\left(l_{s}^{0}, i\right)\right\}\right)}{\sum_{k=0}^{q-1} \exp \left(\frac{1}{T}\left\{\sum_{r \in N_{S}} K_{k k}^{r s}\left[P\left(l_{r}=k\right)\right]^{\text {old }}+B \delta\left(l_{s}^{0}, k\right)\right\}\right)}
\end{aligned}
$$

$\forall i \in \mathcal{I}, \forall s \in S$

where $P\left(l_{s}=i\right)$ is the probability for the label of the site $s$ to be at the state $i, K_{i i}^{r s}$ are the potential coefficients as elements of a diagonal matrix (in image processing plays the role of a smoothness factor), $B$ is the external field (here plays the role of a roughness factor) and $l_{s}^{0}$ is the initial label of the site $s$, new refers to instant $t+1$ and old to instant $t$.

In the simplest case we take $K$ as a scalar and we have: 

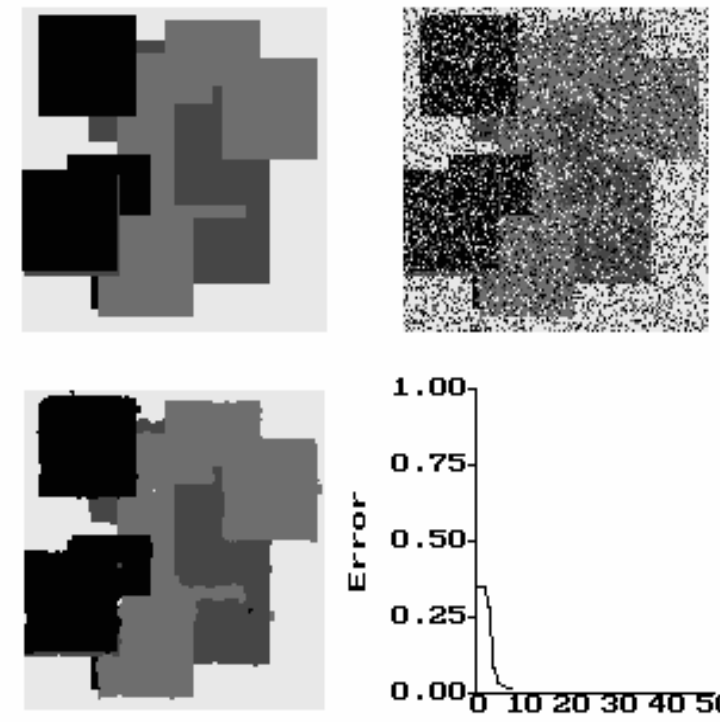

$\mathbf{k}=\mathbf{8} \quad \mathbf{T}=0.500$

Error $=0.015$

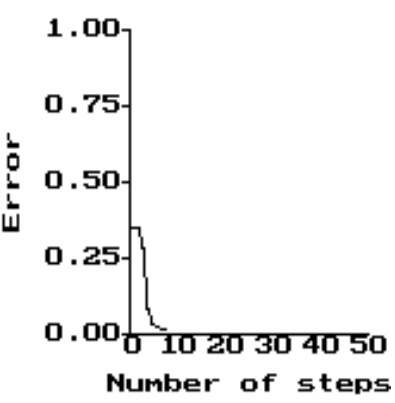

Fig. 5. Results of MFFA after a misclassification of $35 \%$ of pixels by adding uniform channel noise on this image $(128 \times 128)$ with $q=4, K=1.0$ and $B=1.0$. The number of sweeps is $k=8$. Up Left: Artificial image. Up Right: Degraded channel noisy image. Down Left: Relaxation result. Down Right: Classification error as number of steps

$$
\begin{aligned}
& {\left[P\left(l_{s}=i\right)\right]^{\text {new }}} \\
& =\frac{\exp \left(\frac{1}{T}\left\{K \sum_{r \in N_{s}}\left[P\left(l_{r}=i\right)\right]^{\text {old }}+B \delta\left(l_{s}^{0}, i\right)\right\}\right)}{\sum_{k=0}^{q-1} \exp \left(\frac{1}{T}\left\{K \sum_{r \in N_{s}}\left[P\left(l_{r}=k\right)\right]^{\text {old }}+B \delta\left(l_{s}^{0}, k\right)\right\}\right)} \\
& \forall i \in \mathcal{I}, \forall s \in S .
\end{aligned}
$$

\subsection{Label changes and critical temperature}

Combinatorial optimization problems having multiple conflicting constraints are called ill-posed or nonconvex. Nonconvex problems typically have numerous suboptimal solutions manifested as local minima in the energy function. The powerful tool of simulated annealing, which, in theory, eventually converges to an optimal solution, has been applied to a variety of nonconvex combinatorial optimization problems. The control parameter $T$ takes an initial value $T_{\mathrm{o}}$. The deterministic nature of mean-field method acts on the system so that the major part of label changes occur in a small temperature region below critical temperature $T_{\mathrm{c}}$. The temperature $T$ here is a parameter that reduced following a cooling schedule. Thus it's enough to start mean field annealing at some temperature

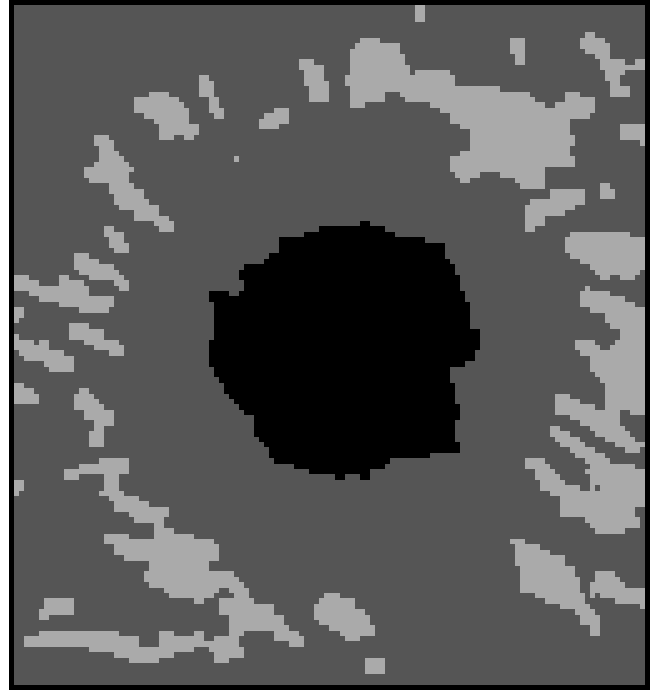

Fig. 6. Sunspot image $(128 \times 128)$ of Fig. 3 after MFFA application. The labels now are three. Labels of too small isolated areas are disappeared

$T_{\mathrm{o}}$ a little higher than $T_{\mathrm{c}}$ in order to reach convergence. There is no link between the parameters $T_{\mathrm{o}}$ and $T_{\mathrm{c}}$ and the measured solar temperatures.

Simulated annealing algorithm reaches an optimal configuration the same way the annealing process of a solid does into its (globally) lowest energy state. In Conventional Simulated Annealing (Geman \& Geman 1984) there is an initial temperature $T_{\mathrm{o}}$ such that at step $k$ we have $T(k)=\frac{T_{\mathrm{o}}}{\log (k)}$. A similar technique developed by Szu \& Hartley (1987) employs the following Fast Cooling Schedule: $T(k)=\frac{T_{\mathrm{o}}}{k}$. Both temperature schedules have been tested within our deterministic frame, leading to very similar results (Bratsolis \& Sigelle 1997). The composition of probabilistic mean-field approximation with the fast cooling schedule gives the following Mean Field Fast Annealing (MFFA) algorithm.

\subsection{The MFFA algorithm}

This algorithm propose a relaxation labeling process.

MFFA algorithm (with $K$ as a scalar), (Figs. 5, 6, 7). 1 . Define $k_{\text {limit }}$ : number of sweeps, and $T_{\mathrm{o}}$ : initial temperature.

2. Initialize $q$ buffers of size $N$ with $P\left(l_{s}=i\right)=10^{-5} \forall i \in$ $\mathcal{I}, \forall s \in \mathcal{S}$.

3. For $k=1 \ldots k_{\text {limit }} T=\frac{T_{\mathrm{o}}}{k}$ Use [Eq. (9)].

4. If $k=k_{\text {limit }}$ Round off and display taking $l_{s}=$ $\arg \max _{i \in \mathcal{I}} P\left(l_{s}=i\right)$.

Taking $K=1.0$ and $B=1.0$ in four-connectivity, i.e., the neighborhood of the site $s$ contains the four nearest 


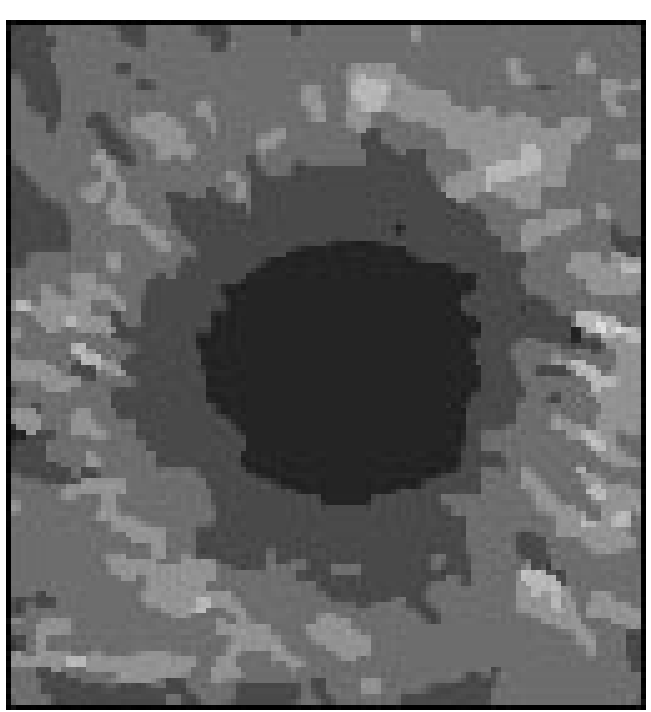

Fig. 7. Sunspot image $(128 \times 128)$ of Fig. 4 after MFFA application. The labels now are six. Labels of too small isolated areas are disappeared

pixels, experimental tests give (Bratsolis \& Sigelle 1997) for $q=4, T_{\mathrm{c}}=1.92$ and for $q=8, T_{\mathrm{c}}=1.36$. In any case we accept $T_{\mathrm{o}} \simeq 2 T_{\mathrm{c}}$.

\section{Results and discussion}

We want to quantize an image into nicely connected areas, so that isolated pixels, or small isolated areas with a gray level different from their background can be eliminated.

As far as dynamics is concerned, we choose a serial mode in which the local field, in each site, is computed immediately before the corresponding site state is updated. We use raster examination, starting at each sweep from the upper left image pixel and finishing with the lower one. When the system converges we display, taking for every site $s$, the maximal probability from every state $q$.

With $N$ sites and $q$ label values (gray levels), MFFA needs $O(q N)$ updates at a fixed temperature while the stochastic Simulated Annealing requires $O\left[\left(q^{N}\right)^{2}\right]$ such steps (Acton \& Bovik 1996; Bratsolis \& Sigelle 1997).

$\mathrm{H}_{\alpha}$ images are usually so complicated that a simple histogram modification is not sufficient to separate the regions of different activity. In this paper we have presented a method of combinatorial optimization to separate our image in different classes. Previous methods used in photospheric images (e.g. Steinegger et al. 1997), separate the images in three classes - umbra, penumbra and photospheric background - for the measure of sunspot areas.
Here we have described how MFFA can be used as a method of segmentation of images previously classified in any number of classes. We have considered a synthetic example in which degradation arose through the simple superposition of uniform label channel noise. After a small number of sweeps (eight in our case) we obtain very good results.

The importance of solar image segmentation is evident because the knowledge of the spatial (on the solar disk) and time variability of sunspots, faculae, network and quiet-Sun is essential to understand the solar cycle (Steinegger et al. 1996). The segmentations of solar images could be considered as a first stage of automated recognition and classification of sunspots, something that we intend to investigate in future work.

Acknowledgements. The authors are grateful to Dr. A. Georgakilas of the Astronomical Institute of National Observatory of Athens for the sunspot image and Dr. T. Roudier for his critical review and suggestions to improve the paper.

\section{References}

Acton S.T., Bovik A.C., 1996, IEEE Trans. Neural Networks 7,686

Besag J., 1974, J. R. Statist. Soc. B 36, 192

Besag J., 1986, J. R. Statist. Soc. B 48, 259

Bilbro G.L, Snyder W.E, Mann R.C., 1991, J. Opt. Soc. Am. A 8,290

Bratsolis E., Sigelle M., 1997, J. Opt. Soc. Am. A 14, 1033

Carnevali P., Coletti L., Patarnello S., 1985, IBM J. Res. Develop. 29, 569

Collados M., Martínez Pillet V., Ruiz Cobo B., del Toro Iniesta J.C., Vázquez M., 1994, A\&A 291, 622

Fröhlich C., Pap J., 1989, A\&A 220, 272

Geman S., Geman D, 1984, IEEE Trans. Patt. Anal. Mach. Int. 6,721

Hérault L., Horaud R, 1993, Trans. Patt. Anal. Mach. Int. 15, 899

Hiriyannaiah H.P., Bilbro G.L, Snyder W.E, Mann R.C, 1989, J. Opt. Soc. Am. A 6, 1901

Hummel R., Zucker S.W., 1983, IEEE Trans. Patt. Anal. Mach. Int. 5, 286

Jeffrey W., Rosner R., 1986, ApJ 310, 473

Pap J., 1985, Solar Phys. 97, 21

Steinegger M., Vázquez M., Bonet J.A., Brandt P.N., 1996, ApJ 461, 478

Steinegger M., Bonet J.A., Vázquez M., 1997, Solar Phys. 171, 303

Szu H.H., Hartley R.L., 1987, Proc. IEEE 75, 1538

Titterington D.M., 1985, A\&A 144, 381

Wu F.Y., 1982, Rev. Mod. Phys. 54, 235 